\section{RSP}

http://www.rsp.fsp.usp.br/
Revista de Saúde Pública

\title{
Tendência temporal da incidência de tuberculose e sua distribuição espacial em Macapá-AP
}

\author{
Clóvis Luciano Giacomet' ${ }^{1}$ iD, Marcio Souza Santos' ${ }^{1}$ iD, Thaís Zamboni Berra' ${ }^{1}$, Yan Mathias \\ Alves' $^{1}$ (D), Luana Seles Alves' (iD), Fernanda Bruzadelli Paulino da Costa' iD, Antonio Carlos \\ Vieira Ramos' (iD, Juliane de Almeida Crispim' (iD, Aline Aparecida Monroe' iD, lone Carvalho

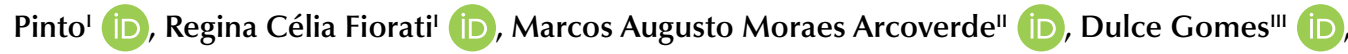 \\ Giselle Lima de Freitas $^{\text {IV }}$ (iD, Mellina Yamamurav ${ }^{\mathrm{V}}$ (iD, Ricardo Alexandre Arcêncio' iD \\ ' Universidade de São Paulo. Escola de Enfermagem de Ribeirão Preto. Ribeirão Preto, SP, Brasil \\ " Universidade do Oeste do Paraná, Escola de Enfermagem. Foz do Iguaçu, PR, Brasil \\ III Universidade de Évora. Departamento de Matemática. Évora, Portugal \\ Iv Universidades Federal de Minas Gerais. Faculdades de Enfermagem. Belo Horizonte, MG, Brasil \\ $\checkmark$ Universidades Federal de São Carlos. Faculdades de Enfermagem. São Carlos, SP, Brasil
}

Correspondência:

Clóvis Luciano Giacomet Travessa Pinheiro Machado, 25, apto 503

85505-060 Pato Branco, Paraná Brasil

E-mail: clovisgiacomet@gmail.com

Recebido: 21 dec 2020

Aprovado: 19 mar 2021

Como citar: Giacomet $\mathrm{CL}$, Santos MZ, Berra TZ, Alves YM, Seles L, Costa FBP, et al. Tendência temporal da incidência de tuberculose e sua distribuição espacial em Macapá-AP. Rev Saude Publica. 2021;55:96. https://doi.org/10.11606/s15188787.2021055003431

Copyright: Este é um artigo de acesso aberto distribuído sob os termos da Licença de Atribuição Creative Commons, que permite uso irrestrito, distribuição e reprodução em qualquer meio, desde que o autor e a fonte originais sejam creditados.

\section{RESUMO}

OBJETIVO: Avaliar a tendência temporal da incidência da tuberculose após a implementação do teste rápido molecular, identificar se a tuberculose apresenta variação sazonal e classificar o território de acordo com a densidade de casos e as áreas de risco em Macapá-AP.

MÉTODOS: Estudo ecológico composto por casos de tuberculose registrados no SINAN entre 2001 e 2017. Foi utilizado o teste Prais-Winsten para classificar a tendência temporal da incidência e a Série Temporal Interrompida para identificar mudanças na tendência temporal antes e depois da implementação do teste rápido molecular, além de verificar a sazonalidade no município. Utilizou-se o estimador de Kernel para classificar a densidade de casos e estatística de varredura para identificar áreas de risco da tuberculose.

RESULTADOS: Foram identificados 1730 casos, observando-se que a tendência temporal da incidência de tuberculose foi decrescente (-0,27\% por mês, IC95\% -0,13 a -0,41). Não houve mudança de nível na série temporal após a implantação do teste molecular GeneXpert ${ }^{\circledR}$ MTB/RIF, porém, o período pós teste foi classificado como crescente em termos da incidência $(+2,09 \%$ por mês, IC95\% 0,92 a 3,27). Quanto à variação sazonal, apresentou crescimento (+13,7\%/mês, IC95\% 4,71 a 23,87) nos meses de dezembro a junho, referente ao período de chuvas - chamado inverno amazônico - e decréscimo (-9,21\% por mês, IC95\% -1,37 a -16,63) nos demais períodos. Por meio de Kernel, foram classificadas áreas com alta densidade de casos nos distritos Central e Norte e, com a estatística de varredura, foram identificados três aglomerados de proteção, AE1 ( $R R=0,07), A E 2(R R=0,23)$ e AE3 ( $R R=0,36)$, e um aglomerado de alto risco, $\mathrm{AE} 4(\mathrm{RR}=1,47)$.

CONCLUSÃO: A tendência temporal da incidência de tuberculose se revelou decrescente na série temporal, todavia, um crescimento na detecção foi observado após introdução do TRM-TB, e ainda se evidenciou que há comportamento sazonal da tuberculose. A distribuição dos casos foi heterogênea, com tendência de concentração em territórios vulneráveis e de risco, evidenciando um padrão de desigualdade da doença no território.

DESCRITORES: Tuberculose, epidemiologia. Conglomerados Espaço-Temporais. Análise Espacial. Estudos Ecológicos. 


\section{INTRODUÇÃO}

A tuberculose permanece como um problema de saúde pública mundial. Segundo a Organização Mundial da Saúde(OMS), é uma das 10 principais causas de morte no mundo e a principal causa de morte por um único agente infeccioso em pessoas vivendo com o vírus da imunodeficiência humana (PVHIV). Em 2019, cerca de 10 milhões de pessoas adoeceram por tuberculose no mundo e cerca de 1,4 milhões de pessoas morreram em decorrência da doença1.

No Brasil, em 2019, foram diagnosticados 73.864 casos novos de tuberculose (incidência de 35,0 casos/100 mil habitantes) e vale destacar que a região Norte dos pais é a mais acometida pela doença². No Amapá, em 2019, foram diagnosticados 296 casos novos da doença distribuídos em 14 dos 16 municípios que compõem o estado, sendo Macapá a cidade com maior número de casos, que registrou um aumento de $23,9 \%$ em relação a $2018^{3}$.

Para sua eliminação, são necessárias ações de maior amplitude, como a busca ativa de sintomáticos respiratórios visando ao diagnóstico precoce de casos e ao início rápido do tratamento, de modo a evitar que a pessoa com tuberculose permaneça na fase bacilífera e transmita a doença para outras pessoas e comunidades ${ }^{4}$.

No que tange ao diagnóstico, o teste rápido molecular para tuberculose (TRM-TB), realizado através do sistema GeneXpert ${ }^{\circledR} \mathrm{MTB} / \mathrm{RIF}$, consiste em um exame automatizado, que reduz o tempo de resultado do diagnóstico para no máximo 2 horas e gera economia nos custos, incluindo aqueles decorrentes de internações resultantes da evolução da doença e do diagnóstico tardio. Os custos de internação devido à tuberculose elevam em até cem vezes com relação àqueles de tratamento ocorrido em nível ambulatorial ${ }^{5}$.

Com resultados mais rápidos e apresentando alta sensibilidade e especificidade, respectivamente de $98 \%$ e $88 \%^{5}$, o tratamento pode ser iniciado precocemente, sem a necessidade de aguardar a confirmação pela cultura (que pode demorar até 60 dias).

A aplicação do TRM-TB ocorreu após a validação de seus resultados pela OMS, em 2010, quando a tecnologia diagnóstica passou a ser recomendada e adotada em vários sistemas de saúde em todo o mundo. No Brasil, a Comissão Nacional de Incorporação de Tecnologias do SUS (CONITEC-SUS) e a Agência Nacional de Vigilância Sanitária (ANVISA) aprovaram a utilização do teste no ano de 2013, quando foram adquiridos 160 equipamentos do laboratório Cepheid pelo Ministério da Saúde, distribuídos estrategicamente pelo país ${ }^{5}$.

Levanta-se a hipótese de que o TRM-TB, por sua alta sensibilidade, modificou o número de casos diagnosticados e contribuiu com a elucidação de casos, especificamente dos paucibacilares, antes não detectados pela baciloscopia. Ou seja, investiga-se se o TRM-TB pode ter mudado o panorama epidemiológico da tuberculose.

Além da elevação de casos, tem-se como outra hipótese do estudo a variação sazonal da doença, fenômeno que tem sido observado em estudos realizados em vários países ${ }^{6}$, todavia pouco explorado no Brasil. Outro estudo ${ }^{7}$ evidenciou a ocorrência de elevação das doenças respiratórias na Amazônia nos períodos de inverno, quando aumentam as chuvas na região e, por isso, levanta-se a hipótese de que esse aumento nos índices possa acontecer também com a tuberculose.

Diante do exposto, o estudo buscou avaliar a tendência temporal da incidência da tuberculose após a implementação do TRM-TB, identificar se ela apresenta variação sazonal e classificar o território de acordo com densidade de casos e áreas de risco para a doença em município da Amazônia Oriental.

\section{MÉTODOS}

\section{Delineamento do Estudo}

Estudo ecológico ${ }^{8}$ realizado em Macapá, capital do estado do Amapá, que está inserido na Amazônia Oriental e faz divisa com a Guiana Francesa. 


\section{Local de Estudo}

A cidade de Macapá conta com uma área de aproximadamente $6.563,849 \mathrm{~km}^{2}$, densidade demográfica de $62,14 \mathrm{hab} / \mathrm{km}^{2}$ e população estimada de 512.902 pessoas em 2020. Está administrativamente dividida em quatro distritos: Norte, Oeste, Centro e Sul ${ }^{9}$.

O diagnóstico dos casos de tuberculose em Macapá segue o protocolo do Ministério da Saúde ${ }^{5}$. Dessa forma, todas as Unidades Básicas de Saúde (UBS) recebem casos suspeitos que, posteriormente, são encaminhados para a unidade de referência do estado, chamada Centro Regional de Doenças Tropicais (CRDT). Trata-se de um serviço centralizado para todo o estado, que realiza acompanhamento dos casos e dispensação dos medicamentos.

O estado conta ainda com o Laboratório Central (LACEN), que realiza os exames laboratoriais, incluindo aqueles para o diagnóstico de tuberculose. No LACEN, encontra-se o único equipamento GeneXpert ${ }^{\circledR}$ MTB/RIF, referência para o diagnóstico de tuberculose, no Amapá. Ressalta-se ainda que a implementação do TRM-TB ocorreu em setembro de 2014, que foi considerado o ano de corte nas séries temporais ${ }^{5}$.

\section{População}

A população do estudo foi composta por casos de tuberculose notificados no Sistema de Notificação de Agravos de Notificação (SINAN) entre 2001 e 2017.

\section{Plano de Análise}

Inicialmente, com o objetivo de caracterizar o perfil dos casos de tuberculose notificados no município em estudo, foram calculadas as frequências absolutas e relativas das variáveis apresentadas na ficha de notificação através do software IBM SPSS Statistics versão 25.

Para classificar a tendência temporal da incidência de tuberculose, a taxa foi calculada de modo mensal, considerando o número absoluto de casos no numerador e a população no denominador, com fator de multiplicação por 1000 habitantes. As taxas de incidência foram logaritmizadas ( $\log 10)$ de modo a estabilizar a variância ao longo do tempo ${ }^{10}$. Os gráficos da distribuição dos casos e das taxas estimadas ao longo da série temporal foram produzidos por meio do software RStudio.

Utilizou-se o método de autorregressão Prais-Winsten através do software STATA para classificar a tendência temporal da incidência de tuberculose crescente, decrescente ou estacionária e, se crescente ou decrescente, foi calculada a porcentagem de variação mensal (MPC - monthly percent change) e seu respectivo intervalo de confiança de 95\% (IC95\%) $)^{10}$.

Com o objetivo de verificar se houve mudança na tendência temporal da incidência de tuberculose após a implementação do TRM-TB e se a doença apresenta variação sazonal, recorreu-se à Série Temporal Interrompida (STI), definida como o mais efetivo recurso para avaliar o impacto de uma intervenção, possibilitando verificar se há influência imediata (mudança de nível) e/ou influência progressiva (mudança de tendência) nos valores da série ${ }^{11}$.

Para aplicação da STI, foram consideradas as taxas de incidência mensais logaritmizadas (Log10) anteriormente calculadas. A mudança de nível foi chamada de "intervenção" e a mudança progressiva, de "pós-intervenção" ${ }^{1}$. Além disso, foram incorporadas nas análises as funções trigonométricas seno e cosseno, de modo a verificar se a tuberculose apresenta variação sazonal ${ }^{10}$. Para a STI, também foi utilizado o software STATA versão 14.

Na primeira etapa, como critérios de inclusão, foram considerados todos os casos notificados de tuberculose de residentes na área urbana do município de Macapá. Para os casos notificados mais de uma vez no sistema, considerou-se a notificação mais atual. É importante destacar 
que, no período analisado, todos os casos arrolados ao estudo são de novas notificações no SINAN e, portanto, foi a incidência estimada.

Para classificar a densidade de casos, recorreu-se ao estimador de densidade de Kernel. Inicialmente, foi necessário realizar a geocodificação dos casos mediante obtenção das coordenadas geográficas (latitude e longitude) dos endereços residenciais dos casos notificados, utilizando a ferramenta Google Earth Pro.

O georreferenciamento dos casos incluídos no estudo foi realizado através do software ArcGis 10.5, considerando os setores censitários urbanos do município de Macapá como unidade de análise e a população residente por setor censitário, como configuração para padronização da análise.

Destaca-se que, na fase da análise espacial, foram excluídos os casos de pessoas em situação de rua, indivíduos privados de liberdade, indígenas ou pessoas cujo endereço de notificação era de órgãos municipais (hospitais ou unidades de saúde) devido à impossibilidade de obtenção das coordenadas geográficas dos endereços residenciais.

Utilizou-se o estimador de intensidade de Kernel, a partir do software ArcGis 10.5, que consiste em um método de interpolação exploratória que define áreas circulares de influência em torno de pontos de ocorrências de um fenômeno, gerando uma densidade superficial para identificação de áreas vulneráveis ${ }^{12}$. Assim, considerando um raio de 1.000 metros $^{13,14}$, os mapas temáticos da distribuição da densidade dos casos de tuberculose também foram gerados no software ArcGIS 10.5.

Por fim, para identificar as áreas de risco para tuberculose em Macapá, foi utilizada a estatística de varredura ${ }^{15}$, em que a procura por aglomerados espaciais é realizada através de um círculo de raio variável em torno do centroide de cada unidade de análise, que, no presente estudo, foram os setores censitários. Na técnica, é calculado o número de casos observados e esperados dentro de cada círculo, procedimento realizado até que todos os centroides sejam testados. Quando o valor observado na área delimitada pelo círculo for maior ou menor do que o esperado, o círculo é chamado de cluster ou aglomerado ${ }^{16}$.

Nessas análises, adotou-se as seguintes características: modelo discreto de Poisson; não existir sobreposição geográfica dos aglomerados; aglomerados com formato circular; 999 replicações na simulação de Monte Carlo; e tamanho da população exposta estipulado pelo coeficiente de Gini, em que o número de casos é comparado aos dados da população de base e o número esperado de casos em cada setor censitário é proporcional ao tamanho da população em risco ${ }^{16,17}$. Além disso, o risco relativo (RR) e intervalo de confiança (IC95\%) de cada aglomerado foram calculados. Destaca-se que foram considerados aglomerados estatisticamente significativos aqueles com $\mathrm{p}<0,05$.

O estudo foi aprovado pelo Comitê de Ética em Pesquisa da Escola de Enfermagem de Ribeirão Preto, com Certificado de Apresentação para Apreciação Ética (CAAE) no 23043019.2.0000.5393.

\section{RESULTADOS}

Entre 2001 e 2017, foram notificados 1.730 casos de tuberculose em Macapá, com idade mínima de um ano e máxima de 89 anos.

Pode-se observar que a maioria dos casos foi do sexo feminino (59,3\%), com idade entre 31 e 59 anos (47,6\%), raça/cor parda (67,9\%) e com ensino fundamental incompleto (46,6\%). Observa-se também a predominância da forma pulmonar $(85,1 \%)$.

Conforme a Tabela 1, observa-se o alto número de informações em branco e/ou ignoradas referente à escolaridade (16,4\%), coinfecção TB-HIV (50\%), TB-Diabetes (41,8\%), alcoolismo 
Tabela 1. Perfil sociodemográfico e clínico-epidemiológico dos casos de tuberculose (TB), notificados em Macapá-AP, Brasil (2001 a 2017).

\begin{tabular}{|c|c|}
\hline Variáveis & n (1.730) (\%) \\
\hline \multicolumn{2}{|l|}{ Idade (anos) } \\
\hline 0 a 14 anos & $74(4,3)$ \\
\hline 15 a 30 anos & $640(37,0)$ \\
\hline 31 a 59 anos & $823(47,6)$ \\
\hline$\geq 60$ anos & $175(10,1)$ \\
\hline Ignorado/branco & $18(1,0)$ \\
\hline \multicolumn{2}{|l|}{ Sexo } \\
\hline Masculino & $704(40,7)$ \\
\hline Feminino & $1.026(59,3)$ \\
\hline \multicolumn{2}{|l|}{ Raça } \\
\hline Branco & $325(18,3)$ \\
\hline Preto & $124(7,7)$ \\
\hline Amarela & $28(1,6)$ \\
\hline Parda & $1.175(67,9)$ \\
\hline Indígena & $14(0,8)$ \\
\hline Ignorado/branco & $64(3,7)$ \\
\hline \multicolumn{2}{|l|}{ Escolaridade } \\
\hline Analfabeto & $128(7,4)$ \\
\hline Ensino fundamental completo & $112(6,5)$ \\
\hline Ensino fundamental incompleto & $803(46,6)$ \\
\hline Ensino médio completo & $224(12,9)$ \\
\hline Ensino superior incompleto & $52(3,0)$ \\
\hline Educação superior completo & $127(7,3)$ \\
\hline Ignorado/branco & $284(16,4)$ \\
\hline \multicolumn{2}{|l|}{ Forma } \\
\hline Extrapulmonar & $236(13,6)$ \\
\hline Pulmonar & $1.472(85,1)$ \\
\hline Pulmonar + Extrapulmonar & $18(1,0)$ \\
\hline Em branco/lgnorado & $4(0,3)$ \\
\hline \multicolumn{2}{|l|}{ Desfecho } \\
\hline Cura & $1.303(75,3)$ \\
\hline Abandono & $217(12,5)$ \\
\hline Óbito por tuberculose & $17(1,0)$ \\
\hline Óbito por outra causa & $47(2,7)$ \\
\hline Transferência/mudança de país & $108(6,3)$ \\
\hline Mudança de diagnóstico & $30(1,7)$ \\
\hline TB multirresistente & $04(0,2)$ \\
\hline Mudança de esquema por intolerância medicamentosa & $03(0,2)$ \\
\hline Mudança de diagnóstico & $01(0,1)$ \\
\hline \multicolumn{2}{|l|}{ Coinfecção TB-HIV } \\
\hline Não & $787(45,5)$ \\
\hline Sim & $77(4,5)$ \\
\hline Ignorado/Em branco & $866(50,0)$ \\
\hline \multicolumn{2}{|l|}{ Coinfecção TB-diabetes } \\
\hline Não & $912(52,8)$ \\
\hline Sim & $94(5,4)$ \\
\hline Ignorado/Em branco & $724(41,8)$ \\
\hline
\end{tabular}


Tabela 1. Perfil sociodemográfico e clínico-epidemiológico dos casos de tuberculose (TB), notificados em MacapáAP, Brasil (2001 a 2017). Continua

\begin{tabular}{lc}
\hline Alcoolismo & $896(51,7)$ \\
Não & $148(8,6)$ \\
Sim & $686(39,7)$ \\
Ignorado/Em branco & $1.001(57,8)$ \\
Doença mental & $12(0,7)$ \\
Não & $717(41,5)$ \\
Sim & $717(0)$ \\
\hline Ignorado/Em branco & \\
\hline
\end{tabular}

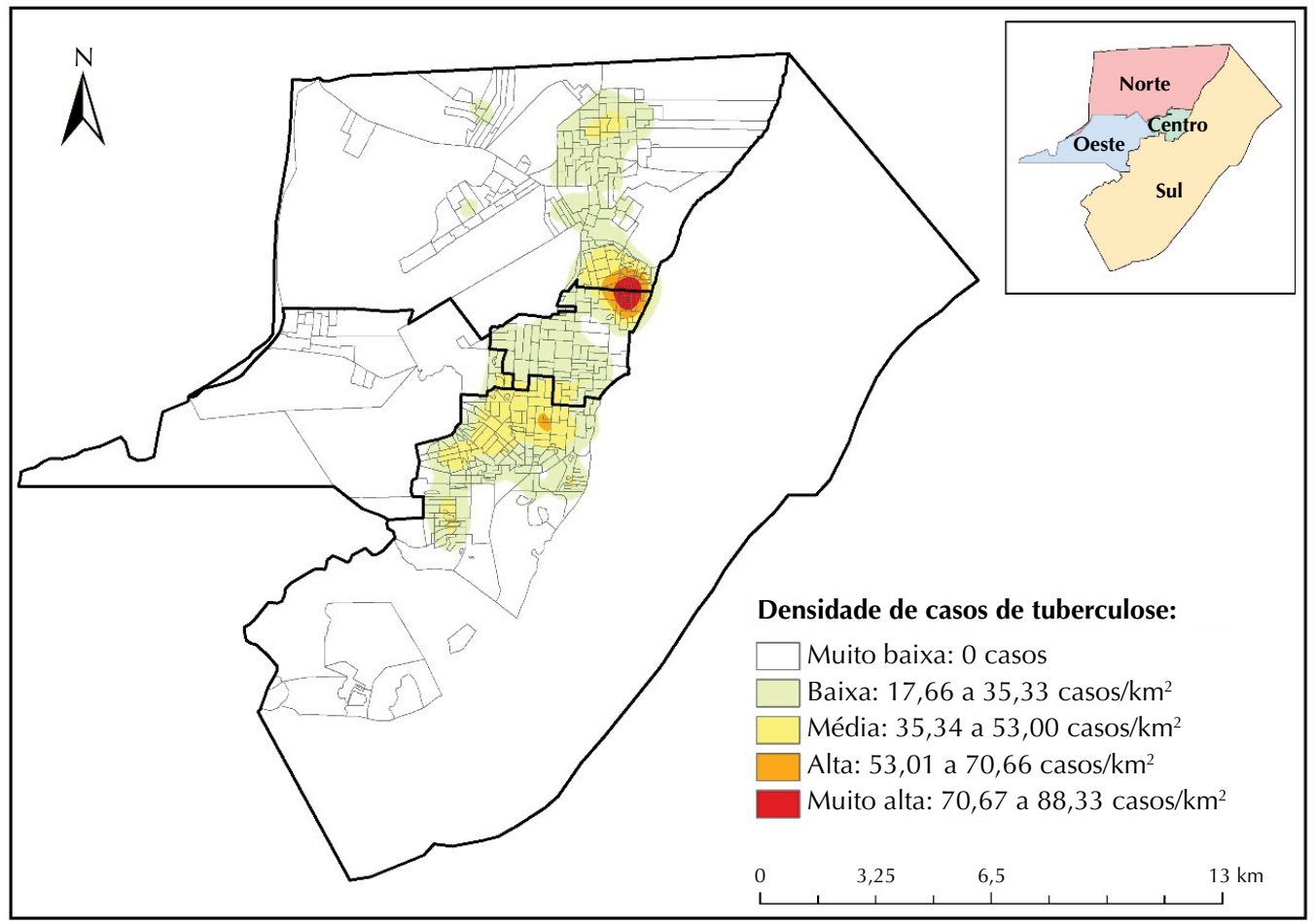

Figura 1. Série temporal do número de casos e da incidência de tuberculose em Macapá-AP, Brasil (2001 a 2017).

(39,7\%) e doença mental (41,7\%). Não obstante, essas informações são relevantes para o manejo clínico dos casos e das condutas.

A Figura 1 apresenta a série temporal do número de casos e da incidência de tuberculose ao longo dos meses de 2001 a 2017, em que é possível observar a presença de picos e quedas por todo o período em estudo. Conclui-se, portanto, que a série inicia-se com números mais elevados, sofre uma queda e finaliza de maneira crescente.

A Tabela 2 apresenta os resultados das técnicas de Prais-Winsten e STI. Foi possível classificar a tendência temporal da incidência de tuberculose em Macapá como decrescente, com queda de $-0.27 \% /$ mês (IC95\% -0.41 a -0.13 ).

Também foi identificado que não houve mudança de nível (intervenção) na série temporal após a implementação do TRM-TB. Todavia, o período pós implantação do TRM-TB foi classificado como crescente, com aumento de 2,09\%/mês (IC95\% 3,27 a 0,92), indicando que houve um aumento progressivo na incidência da tuberculose após a implementação do teste.

Quanto à sazonalidade, foi possível identificar que a doença em Macapá apresenta crescimento de 13,7\%/mês (IC95\% 23,87 a 4,71) nos meses entre dezembro e junho (seno), 
Tabela 2. Tendência temporal, impacto do diagnóstico por meio do teste rápido molecular e variação sazonal na incidência de casos de tuberculose, Macapá - AP, Brasil (2001 a 2017).

\begin{tabular}{|c|c|c|c|}
\hline \multicolumn{4}{|c|}{ Prais-Winsten } \\
\hline & $\begin{array}{l}\text { Coeficiente } \\
\text { (IC95\%) }\end{array}$ & Tendência & $\begin{array}{c}\text { MPC } \\
(\text { IC95\%) }\end{array}$ \\
\hline Tuberculose em Macapá & $\begin{array}{c}-0,001 \\
(-0,006 \text { a }-0,000)\end{array}$ & Decrescente & $\begin{array}{c}-0,27 \\
(-0,41 \text { a }-0,13)\end{array}$ \\
\hline \multicolumn{4}{|c|}{ Série temporal interrompida (STI) } \\
\hline & $\begin{array}{l}\text { Coeficiente } \\
\text { (IC95\%) }\end{array}$ & Tendência & $\begin{array}{c}\text { MPC } \\
(\text { IC95\%) }\end{array}$ \\
\hline Intervenção & $\begin{array}{c}1,014 \\
(3,001 \mathrm{a}-0,971)\end{array}$ & Estacionário & NA \\
\hline Pós-Intervenção & $\begin{array}{c}0,009 \\
(0,014 \text { a } 0,004)\end{array}$ & Crescente & $\begin{array}{c}2,09 \\
(3,27 \text { a } 0,92)\end{array}$ \\
\hline Seno & $\begin{array}{c}0,056 \\
(0,093 \text { a } 0,020)\end{array}$ & Crescente & $\begin{array}{c}13,76 \\
(23,87 \text { a } 4,71)\end{array}$ \\
\hline Cosseno & $\begin{array}{c}-0,042 \\
(-0,079 \text { a }-0,006)\end{array}$ & Decrescente & $\begin{array}{c}-9,21 \\
(-16,63 a-1,37)\end{array}$ \\
\hline
\end{tabular}

MPC: monthly percent change; NA: não se aplica.

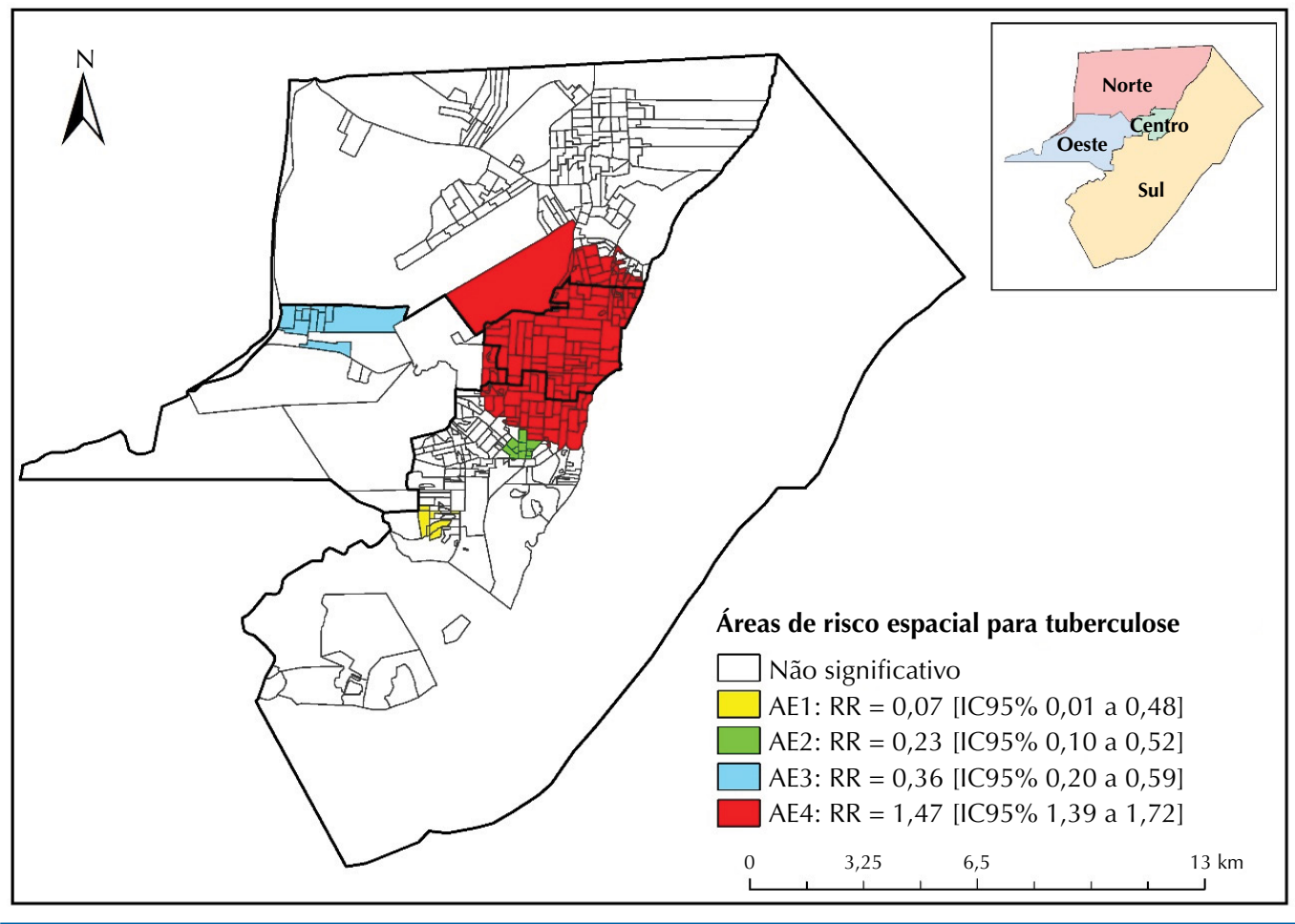

Figura 2. Localização geografia dos casos de tuberculose notificados em Macapá-AP, Brasil (2001 a 2017).

período coincidente com as chuvas ocorridas no chamado inverno amazônico. Outrossim, identificou-se decréscimo de 9,21\%/mês (IC95\% -1,37 a -16,63) nos meses restantes (cosseno), que são épocas mais quentes e de estiagem.

Dos 1730 casos de tuberculose identificados, 59 (3,41\%) foram excluídos por não possuírem endereço. Dos 1.671 casos restantes, $1475(88,2 \%)$ tiveram as coordenadas geográficas identificadas e, portanto, georreferenciadas.

Com o estimador de Kernel, pode-se observar áreas classificadas como muito alta densidade de casos nos distritos Central (bairros Laguinho e Perpétuo Socorro) e Norte (bairros Pacoval e Cidade Nova), com variação de 70,67 a 88,33 casos $/ \mathrm{km}^{2}$. Áreas classificadas como alta densidade de casos estão nos distritos Sul (bairro Buritizal), Centro (bairros Laguinho e Perpétuo Socorro) e Norte (bairros Pacoval e Cidade Nova), com variação de 53,01 a 70,66 casos $/ \mathrm{km}^{2}$, conforme Figura 2. 


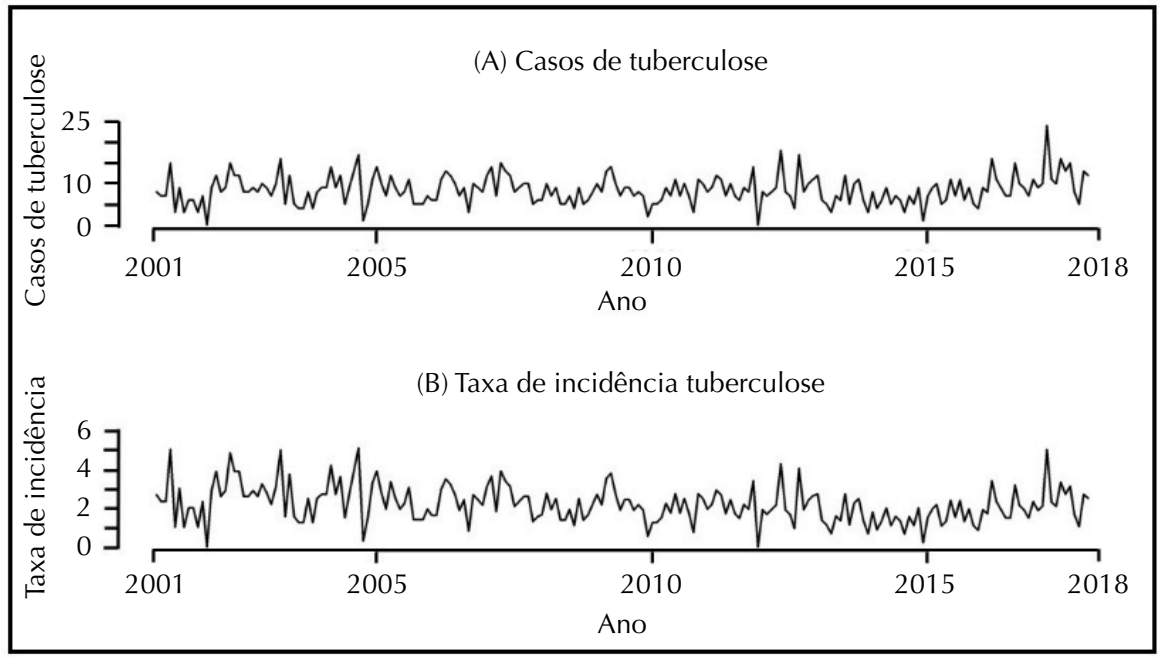

Figura 3. Áreas de risco espacial para tuberculose em Macapá-AP, Brasil (2001 a 2017).

A estatística de varredura possibilitou a identificação de quatro aglomerados espaciais (AE) de risco para a tuberculose em Macapá (Figura 3), considerando o parâmetro de $40 \%$ da população exposta, segundo o índice de Gini.

O AE1, considerado de proteção ( $R R=0,07$; IC95\% 0,01 a 0,48), foi formado por seis setores censitários ( $\mathrm{p}=0,03)$ do distrito Sul, com população de 3.799 habitantes, um caso observado e 14 casos esperados.

O AE2, também de proteção ( $\mathrm{RR}=0,23$; IC95\% 0,10 a 0,52), foi composto por 10 setores censitários $(\mathrm{p}=0,03)$ do distrito Sul, com população de 6.578 habitantes, seis casos observados e 25 casos esperados.

O AE3, também considerado de proteção (RR = 0,36; IC95\% 0,20 a 0,59), foi formado por 11 setores censitários do distrito Oeste $(\mathrm{p}=0,04)$, com 10.172 habitantes, 14 casos observados e 38 casos esperados.

Por fim, o AE4 ( $\mathrm{p}<0,01)$, considerado de risco para a tuberculose $(\mathrm{RR}=1,47$; IC95\% 1,39 a 1,72), foi formado por 146 setores censitários dos distritos Norte, Sul e Centro, com população de 122.356 habitantes, 620 casos observados e 487 casos esperados.

\section{DISCUSSÃO}

Foi possível traçar o perfil sociodemográfico e clínico-epidemiológico dos casos de tuberculose em Macapá-AP. Com o cálculo da incidência mensal numa série temporal de 16 anos, foi possível classificar a tendência temporal e identificar mudanças na série após a implementação do TRM-TB, além de evidenciar que a tuberculose apresenta variação sazonal. Por fim, foi possível classificar áreas de acordo com a densidade de casos e identificar áreas de risco e proteção em Macapá-AP.

A maioria das pessoas acometidas por tuberculose em Macapá era do sexo feminino, resultado diferente dos achados da literatura, haja vista a evidência de que a maioria dos casos geralmente ocorre em pessoas do sexo masculino, evidência explicada pelo fato de que o homem tende a minimizar seu autocuidado e a estar mais exposto aos fatores de risco para a doença ${ }^{18}$.

A predominância do sexo feminino pode estar relacionada ao fato de as mulheres serem chefes de família, subvalorizadas no mercado de trabalho e de desempenharem, grande parte das vezes, tripla jornada de trabalho. O que são fatores estressantes que podem 
ser considerados de risco para o adoecimento ${ }^{18}$. Além disso, destaca-se a desigualdade de gênero, herdada de questões culturais locais dos povos ribeirinhos, em que a mulher é vista como coadjuvante e o homem, como provedor, características ainda presentes no cenário de estudo.

A maioria dos casos foi detectada em pessoas adultas em faixa etária economicamente ativa, fase da vida em que os indivíduos tendem a enfrentar maiores aglomerações, por conta do trabalho e das atividades cotidianas, aumentando as chances de adoecimento ${ }^{19}$.

Pessoas pardas apresentaram mais propensão à doença no estudo, o que pode ser justificado por ser a raça/cor predominante no município. Quase 52\% da população Macapá se autodeclara parda e, neste estudo, os autodeclarados pardos são 67,9\%, o que justificaria os altos índices da tuberculose nessa população.

A maioria dos casos notificados possui ensino fundamental incompleto, que é um indicador indireto de condições sociais acarretadas pelo contexto social no qual a população está inserida. Esse dado representa um entrave para o controle da tuberculose, visto que esses indivíduos poderão ter dificuldades na compreensão da doença e das orientações para seu tratamento e prevenção ${ }^{20}$.

Mediante esse panorama, o profissional de saúde deve estar apto a esclarecer essas situações de formas complementares, buscando maneiras de aumentar a efetividade do controle da tuberculose, bem como uma visão holística do cuidado em saúde ${ }^{21}$.

Também foi observada a predominância da forma clínica pulmonar, o que era esperado, visto que é a forma mais comum da doença ${ }^{1,2}$. A maioria dos casos teve cura (75,3\%), todavia, essa taxa ficou muito abaixo da recomendação da OMS, de $85 \%^{1}$. Os achados acenam para o elevado número de abandonos (12,5\%), acima do estabelecido pela OMS (5\%), que é fator preditor para desenvolvimento da tuberculose resistente, cujo tratamento é mais longo e mais oneroso para o sistema de saúde ${ }^{20}$.

Similarmente, foi evidenciado um alto percentual de variáveis em branco, como as comorbidades de HIV, diabetes, alcoolismo e doenças mentais; essas informações são de suma relevância para o manejo clínico dos casos. Alguns pressupostos para os não-registros são a não priorização dessas informações na clínica, a qualificação incipiente das equipes sobre importância da vigilância em saúde baseada na qualidade dos dados, e a falta de avaliação e devolutiva às equipes sobre os dados produzidos nas unidades nas quais os diagnósticos foram realizados.

No período estudado, a incidência da tuberculose foi decrescente, padrão que segue a tendência mundial ${ }^{1,22}$. Porém, esse declínio ocorre em velocidade aquém das expectativas da $\mathrm{OMS}^{1}$ e, desse modo, é pouco provável que se alcance a eliminação da doença até 2050 .

Com a STI, não foi identificada mudança de nível após a implementação do TRM-TB, porém, o período de "pós-intervenção" foi classificado como crescente, confirmando a hipótese de que o teste, por sua alta sensibilidade ${ }^{23-25}$, possa ter influenciado no aumento de casos no município.

A baciloscopia permanece no SUS como método para acompanhamento mensal do tratamento, sendo o TRM-TB realizado somente para o diagnóstico inicial. A cultura, considerada padrão-ouro, leva em torno de 45 a 60 dias, devido à replicação lenta do bacilo, o que impossibilita ser utilizada como única estratégia para diagnóstico precoce e terapêutica oportuna $a^{4,5}$.

Todavia, o uso do TRM-TB não prescinde o da cultura, que deve ser considerada de forma universal, para que seus dados sejam confrontados com os do TRM-TB (passível de falsos positivos quanto à sensibilidade ou resistência da rifampicina) e, além disso, triar cepas resistentes aos medicamentos ${ }^{24}$. 
A principal estratégia para o controle da tuberculose é a detecção precoce dos casos, principalmente os casos pulmonares bacilíferos, que possuem maior importância epidemiológica, visando à manutenção da cadeia de transmissão da doença, seguida pelo tratamento adequado e alcance da cura ${ }^{26}$. Segundo a $\mathrm{OMS}^{27}$, entre os anos de 2000 e 2014, foi possível evitar o óbito de cerca de 43 milhões de pessoas através de diagnóstico precoce e tratamento adequado. Dessa maneira, entende-se que estratégias e políticas públicas que tenham essa finalidade podem, a médio ou longo prazo, propiciar a diminuição dos índices da doença e, frente a isso, meios confiáveis de diagnóstico são essenciais.

Outra evidência trazida é a sazonalidade da tuberculose, verificando-se oscilações significativas de caso e incidência ao longo do ano, confirmando, portanto, uma das hipóteses do estudo. Os achados evidenciaram que a incidência aumenta entre dezembro e junho, coincidentemente com os períodos de chuvas. Esse comportamento foi observado nas doenças respiratórias na Amazônia Oriental ${ }^{7}$, mas ainda não tinha sido evidenciado no contexto da tuberculose.

Esse fato pode ser atribuído à necessidade de as pessoas concentrarem-se em ambientes superlotados e com pouca ventilação nos períodos em questão. Em contraste a isso, em épocas mais quentes, a tendência temporal apresentou decréscimo, visto que as pessoas procuram ficar em ambientes mais arejados, o que pode diminuir o contágio do bacilo causador da tuberculose.

Com o uso do Kernel e estatística de varredura, foi possível identificar que as maiores densidades de casos e áreas de risco (AE4) estão nos distritos Central, Norte e Sul e nas regiões que concentram os menores Índices de Desenvolvimento Humano do município, que, somados à existência de áreas de ponte (casas de palafita), à ausência de saneamento, aos aglomerados populacionais e ao grande número de trabalhadores informais, em conjunto, corroboram para a disseminação da doença.

Próximo às localidades acima referidas, foram identificadas outras áreas consideradas de proteção (AE1, AE2 e AE3), o que leva à suposição de que há casos não diagnosticados nessas regiões. Portanto, há a possibilidade de subnotificação, visto que a vulnerabilidade social é também marcante nessas regiões. Esse resultado deve ser analisado com a devida cautela e serve de alerta para as equipes de vigilância em saúde.

Considera-se importante a adoção de estratégias como políticas de transferência de renda e/ou compensatórias, visando aliviar a desigualdade social e seus efeitos deletérios na população vulnerável ${ }^{28}$.

Dentre as limitações deste estudo, destaca-se a falácia ecológica ${ }^{29}$, em que os dados são analisados em nível agregado e seus resultados não podem ser interpretados em nível individual. Cabe também mencionar o uso de dados secundários, que podem conter falhas de digitação e informações em branco, que podem interferir nas análises ${ }^{30}$. Outra limitação foi a não existência da variável socioeconômica renda, que poderia ser utilizada como covariável no ajustamento do modelo temporal.

O estudo avança no conhecimento na medida em que se utiliza de duas abordagens distintas para a compreensão do comportamento da tuberculose na Amazônia Oriental, das séries temporais e de análises espaciais. Acresce à sua originalidade a evidência da influência do TRM-TB nas taxas de incidência da tuberculose. Seu ineditismo reside, também, no fato de evidenciar seu comportamento sazonal na Amazônia Oriental, antes não conhecido naquele cenário.

A investigação pode se tornar uma referência para investigações futuras intencionadas na compreensão de agravo. Ajudará, também, na orientação de políticas públicas, haja vista que ficam evidenciadas as diferenças nos territórios em termos da carga da tuberculose, sendo a vulnerabilidade social uma das prováveis explicações para esse cenário. 


\section{REFERÊNCIAS}

1. World Health Organization. Global Tuberculosis Report 2020. Geneva (CH): WHO; 2020 [citado 15 mar 2021]. Disponível em: https://apps.who.int/iris/bitstream/hand le/10665/336069/9789240013131-eng.pdf

2. Ministério da Saúde (BR), Secretaria de Vigilância em Saúde. Bol Epidemiol Tuberculose 2020. 2020 [citado 15 mar 2021]; № Espec:1-40 Disponível em: http://www.aids.gov.br/pt-br/pub/2020/boletim-epidemiologico-de-turbeculose-2020

3. Amapá [Estado], Superintendência de Vigilância em Saúde, Unidade de Doenças Transmissíveis. Bol Epidemiol Tuberculose. 2020 [citado 15 mar 2021];(1). Disponível em: https://editor.amapa. gov.br/arquivos_portais/publicacoes/SVS_c20f41e26fed90da418341d2d2135a3a.pdf

4. Ministério da Saúde (BR), Secretaria de Vigilância em Saúde, Departamento de Vigilância das Doenças Transmissíveis. Manual de recomendações para o controle da tuberculose no Brasil. 2. ed. atual. Brasília, DF: 2019 [citado15 mar 2021]. Disponível em: https://bvsms.saude.gov.br/ bvs/publicacoes/manual_recomendacoes_controle_tuberculose_brasil_2_ed.pdf

5. Ministério da Saúde (BR), Secretaria de Vigilância em Saúde, Departamento de Vigilância das Doenças Transmissíveis. Rede de Teste Rápido para Tuberculose no Brasil: primeiro ano da implantação. Brasília, DF; 2015 [citado 15 mar 2021. Disponível em: http://portalarquivos.saude.gov.br/images/pdf/2016/janeiro/19/rtr-tb-15jan16-isbn-web.pdf

6. Fares A. Seasonality of tuberculosis. J Glob Infect Dis. 2011;3(1):46-55. https://doi.org/10.4103/0974-777X.77296

7. Fundação de Vigilância em Saúde do Amazonas (BR). Situação epidemiológica da Síndrome Respiratória Aguda Grave no Estado do Amazonas. Bol Epidemiol. 2021 [citado 15 mar 2021];8(11). Disponível em: https://www.fvs.am.gov.br/media/publicacao/ Boletim_Epidemiol\%C3\%B3gico_N\%C2\%BA_11.pdf

8. Rothman KJ, Greenland S, Lash TL. Modern epidemiology. 3. ed. Philadelphia, PA: Lippincott Williams \& Wilkins; 2008.

9. Instituto Brasileiro de Geografia e Estatística. Censo 2010: cidades: Macapá. Rio de Janeiro: IBGE; 2016 [citado 15 mar 2021]. Disponível em: https://cidades.ibge.gov.br/brasil/ap/macapa/panorama

10. Antunes JLF, Cardoso MRA. Uso da análise de séries temporais em estudos epidemiológicos. Epidemiol Serv Saude. 2015;24(3):565-76. https://doi.org/10.5123/S1679-49742015000300024

11. Wagner AK, Soumerai SB, Zhang F, Ross-Degnan D. Segmented regression analysis of interrupted time series studies in medication use research. J Clin Pharm Ther. 2002;27(4):299-309. https://doi.org/10.1046/j.1365-2710.2002.00430.x

12. Environmental Systems Research Institute. ArcGIS for Desktop. How Kernel Density works. West Redlands, CA: Esri; c2016 [citado15 mar 2021]. Disponível em: https://desktop.arcgis.com/en/ arcmap/10.3/tools/spatial-analyst-toolbox/how-kernel-density-works.htm

13. Oliveira U, Brescovit AD, Santos AJ. Delimiting areas of endemism through Kernel Interpolation. PLoS One. 2015;10(1):e0116673. https://doi.org/10.1371/journal.pone.0116673

14. Yamamura M, Freitas IM, Santos-Neto M, Chiaravalloti-Neto F, Popolin MAP, Arroyo LH, et al. Análise espacial das internações evitáveis por tuberculose em Ribeirão Preto, SP (2006-2012). Rev Saude Publica. 2016;50:20. https://doi.org/10.1590/S1518-8787.2016050006049

15. Kulldorff $M$, Nagarwalla N. Spatial disease clusters: detection and inference. Stat Med. 1995;14(8):799-810. https://doi.org/10.1002/sim.4780140809

16. Kulldorff M. SaTScan ${ }^{T M}$ manual do usuário para versão 9.4: versão do original traduzido para o Português. Pelini, ACG tradutor. São Paulo; 2016 [citado 15 mar 2021]. Disponível em: https://www.satscan.org/SaTScan_TM_Manual_do_Usu\%C3\%A1rio_v9.4_Portugues.pdf

17. Han J, Zhu L, Kulldorff M, Hostovich S, Stinchcomb DG, Tatalovich Z, et al. Using Gini coefficient to determining optimal cluster reporting sizes for spatial scan statistics. Int J Health Geogr. 2016;15(1):27. https://doi.org/10.1186/s12942-016-0056-6

18. Hoshino $\mathrm{H}$, Uchimura K, Yamauchi Y. [Comparison of TB incidence of young and middle age groups between urban/suburban prefectures and other prefectures]. Kekkaku. 2009;84(1):1-8. Japanese. https://doi.org/10.11400/kekkaku.84.1

19. Fontes GJF, Silva TG, Sousa JCM, Feitosa ANA, Silva ML, Bezerra ALD, et al. Perfil epidemiológico da tuberculose no Brasil no período de 2012 a 2016. Rev Bras Educ Saude. 2019;9(1):19-26. https://doi.org/10.18378/rebes.v9i1.6376 
20. Santos JN, Sales CMM, Prado TN, Maciel EL. Fatores associados à cura no tratamento da tuberculose no estado do Rio de Janeiro, 2011-2014. Epidemiol Serv Saude. 2018;27(3):e2017464. https://doi.org/10.5123/s1679-49742018000300015

21. Coriolano-Marinus MWL, Queiroga BAM, Ruiz-Moreno L, Lima LS. Comunicação nas práticas em saúde: revisão integrativa da literatura. Saude Soc. 2014;23(4):1356-69. https://doi.org/10.1590/S0104-12902014000400019

22. World Health Organization. Global Tuberculosis Report 2019. Geneva (CH): WHO; 2019 [citado 15 mar 2021]. Disponível em: https://apps.who.int/iris/bitstream/hand le/10665/329368/9789241565714-eng.pdf

23. Boehme CC, Nabeta P, Hillemann D, Nicol MP, Shenai S, Krapp F, et al. Rapid molecular detection of tuberculosis and rifampin resistance. N Engl J Med. 2010;363(11):1005-15. https://doi.org/10.1056/NEJMoa0907847

24. Lima TM, Belotti NCU, Nardi SMT, Pedro HSP. Teste rápido molecular GeneXpert MTB/RIF para diagnóstico da tuberculose. Rev Pan Amaz Saude 2017;8(2):67-78.

25. Pandey P, Pant ND, Rijal KR, Shrestha B, Kattel S, Banjara MR, et al. Diagnostic accuracy of GeneXpert MTB/RIF assay in comparison to conventional drug susceptibility testing method for the diagnosis of multidrug-resistant tuberculosis. PLoS One. 2017;12(1):e0169798. https://doi.org/10.1371/journal.pone.0169798

26. Spagnolo LML, Tomberg JO, Vieira DA, Gonzales RIC. Detecção da tuberculose: fluxo dos sintomáticos respiratórios e resultados alcançados. Rev Bras Enferm. 2018;71(5):2543-51. https://doi.org/10.1590/0034-7167-2017-0457

27. World Health Organization. Global Tuberculosis Report 2016. Geneva (CH): WHO; 2019 [citado 15 mar 2021]. Disponível em: https://apps.who.int/iris/bitstream/hand le/10665/250441/9789241565394-eng.pdf?sequence=1\&isAllowed=y

28. Moreira ASR, Kinski AL, Carvalho ACC. Social determinants of health and catastrophic costs associated with the diagnosis and treatment of tuberculosis. J Bras Pneumol. 2020;46(5):e20200015. https://doi.org/10.36416/1806-3756/e20200015

29. Veras RP, Almeida Filho N, Barreto ML, Veras RP, Barata RB. Teoria epidemiológica hoje: fundamentos, interfaces, tendências. Rio de Janeiro: Editora FIOCRUZ; 1998 [citado 15 mar 2021]. (Séries Epidemiológicas, № 2). Disponível em: http://books.scielo.org/id/5btwk

30. Melo GBT, Valongueiro S. Incompleteness of Mortality Information System records on deaths from external causes in Pernambuco, Brazil, 2000-2002 and 2008-2010. Epidemiol Serv Saude. 2015;24(4):651-60. https://doi.org/10.5123/S1679-4974201500040000

Financiamento: Fundação de Amparo à Pesquisa do Estado de São Paulo (Fapesp - Bolsa doutorado Processo no 2018/03700-7). Bolsa Produtividade em Pesquisa Conselho Nacional de Desenvolvimento Científico e Tecnológico (CNPq - Processo 304483/2018-4 - Modalidade PQ - Nível 1C). Coordenação de Aperfeicoamento de Pessoal de Nível Superior (Capes - Código de Financiamento 001 - Programa PROEX - Auxílio № 0524/2018, No Processo: 23038.003643/2018-11).

Contribuição dos Autores: Concepção e planejamento do estudo: CLG, TZB. Coleta, análise e interpretação dos dados: CLG, TZB, MZS. Elaboração ou revisão do manuscrito: CLG, TZB, LS, FBPC, ACVR, YMA, MAMA, JAC, AM, IC, RCF, RAG, MY. Aprovação da versão final: CLZ, TZB, ACVR, RAA. Responsabilidade pública pelo conteúdo do artigo: CLZ, TZB.

Conflito de Interesses: Os autores declaram não haver conflito de interesses. 